\title{
Razonamiento estadístico en estudiantes de quinto de primaria a partir de predicciones en torno a la idea de resistencia física ${ }^{1}$
}

\author{
Statistical Reasoning in fifth-grade students from \\ predictions about the idea of physical endurance \\ Raciocínio estatístico em estudantes de quinta série de \\ previsões sobre a idéia de resistência física
}

Recibido: mayo de 2013

Aceptado: agosto de 2013
Erika Liseth Hernández Hernández ${ }^{2}$ Jhonathan Cuevas ${ }^{3}$

\section{Resumen}

Es lugar común en la comunidad de educadores matemáticos el interés por el desarrollo de competencias que les permitan a los estudiantes comprender, representary predecir una situación o fenómeno a partir de relaciones presentes entre las variables que lo constituyen. En este documento se reportan los resultados de una secuencia de actividades que tiene por finalidad potenciar el desarrollo de razonamiento estadístico en estudiantes de quinto de primaria como medio para la construcción de predicciones respecto a la resistencia física..

Palabras clave: Matemáticas escolares; estadísticas; predicción; aprendizaje; procesos cognitivos; razonamiento; razonamiento estadístico.

\begin{abstract}
It is common place in the community of mathematics educators interest in the development of skills that enable students to understand, represent and predict a situation or phenomenon from present relationships between variables that constitute it. In this paper we report the results of a sequence of activities that aims at improving the development of statistical thinking in fifth-grade students as a means to build predictions about endurance..
\end{abstract}

Keywords: School mathematics, statistics, prediction, learning, cognitive processes, reasoning, statistical reasoning.

\section{Resumo}

É lugar comum na comunidade de matemática educadores interesse no desenvolvimento de competências que permitam aos estudantes entender,

1 Artículo de Investigación.

2 Universidad Distrital Francisco José de Caldas. Bogotá, Colombia. Contacto: erika-col@hotmail.com

3 Universidad Distrital Francisco José de Caldas. Bogotá, Colombia. Contacto: jhonathan_2589@hotmail.com 
representar e prever uma situação ou fenômeno das atuais relações entre as variáveis que o constituem. Neste artigo apresentamos os resultados de uma seqüência de atividades que visa melhorar o desenvolvimento do pensamento estatístico nos alunos da quinta série, como um meio para construir previsões sobre a resistência.

Palavras-chave: A matemática escolar, estatísticas, previsão, de aprendizagem, processos cognitivos, o raciocínio, o raciocínio estatístico..

\section{Contextualización}

Reconociendo como interés continuo en las matemáticas escolares, la puesta en escena de actividades que le permitan a los estudiantes desarrollar razonamiento estadístico (MEN, 1998),y al considerar que especialmente en los primeros años de escolaridad, este tipo de razonamiento se encuentra apoyado en contextos y materiales físicos (MEN, 2006),resulta pertinente diseñar ambientes que involucren a los estudiantes en el análisis de situaciones que permitan por medio de la interacción, el uso de representaciones y su interpretación, predecir el comportamiento de un fenómeno.

Es por ello que en el marco del curso práctica intermedia V, planteado para estudiantes a profesor de Matemáticas en la universidad Distrital Francisco José de Caldas, durante el segundo semestre del año 2012,se llevó a cabo una propuesta de trabajo con noventa estudiantes de grado quinto del Colegio técnico Jaime Pardo leal, que considerara dos elementos fundamentales, el primero, ligado a la idea de razonamiento estadístico como un proceso visto a partir de los planteamientos de Wild \& Pfannkuch (citados por Batanero, 2003) y un segundo elemento de naturaleza metodológica ligado a la teoría de situaciones didácticas (TSD), ya que dicho modelo permite pensar la enseñanza de las matemáticas como un proceso centrado en la producción de conocimiento que realiza el estudiante a partir de sus experiencias y de su entorno como un factor de contradicciones, dificultades y desequilibrios (Panizza,2004).

\section{Referentes teórico-prácticos básicos}

Considerando la predicción como la acción de utilizar una regla o principio para anticipar un resultado o inferir alguna consecuencia, autores como Wild \& Pfannkuch (citados por Batanero, 2003) consideran que dicha acción implica un razonamiento particular, denominado razonamiento estadístico el cual incluye componentes fundamentales, los cuales son:

Reconocer la necesidad de los datos. Refiere a que el análisis de un fenómeno de la vida real sólo puede comprenderse desde el estudio de datos que han sido recogidos en forma adecuada, lo cual de manera directa implica la experimentación. De éste modo si el fenómeno a estudiar es resistencia física, resulta fundamental que los estudiantes pongan en juego el desarrollo de actividades que impliquen esfuerzo físico que a su vez, pueda ser sistematizado.

Transnumeración. Según Batanero (2003) los autores usan este término para indicar la comprensión que puede surgir al cambiar la representación de los datos, la cual está caracterizada a partir de tres tipos, el primero es capturar las características del mundo real, el segundo pasar de los datos iníciales a una representación tabular o gráfica y el tercero corresponde a comunicar el significado que resulta de los datos recolectados.

Se considera que el paso fundamental en esta etapa está localizado en la transformación de la experimentación a una representación tabular o gráfica, pues ello implica desde el fenómeno particular que aquí se estudia entender por ejemplo, que la resistencia física puede estar mediada principalmente por variables como el tiempo y la actividad física realizada.

Razonamiento con modelos estadísticos. Hace referencia al uso de gráficos simples que al ser 
representativos de cierta realidad se constituyen en un modelo.

Ahora bien, retomando los componentes fundamentales del razonamiento estadístico y en particular la percepción de la variación, Arellano (2001) expone que la exploración de los patrones de datos y la selección de técnicas de pronóstico pueden ser de naturaleza cuantitativa y/o cualitativa lo cual depende de ciertas premisas sobre la realidad. En el caso de La técnica cuantitativa se presume que el pasado puede extenderse en el futuro y por ende, la idea de tendencia se encuentra inmersa en dicha visión. Ésta idea junto con la fase de transnumeración mencionada anteriormente, implica que las representaciones tabulares y gráficas cartesianas deben relacionar los factores decisivos que estén involucrados durante la ejecución de un esfuerzo físico, con el tiempo como variable condicionada para ésta técnica de pronóstico.

\section{Descripción general de la propuesta de aula}

Considerando los componentes fundamentales del razonamiento estadístico y las fases dispuestas desde la TSD, la cual propone como punto de partida construir una situación que permita a los estudiantes adquirir un saber determinado (Panizza, 2004), se planteó a los alumnos la siguiente situación fundamental:

La resistencia física es la capacidad de mantener un esfuerzo eficaz durante el mayor período de tiempo posible. Tomando en cuenta la anterior información, ¿Qué estudiantes del grado quinto del colegio técnico Jaime Pardo Leal, tienen mayor resistencia física?

Con el ánimo de desarrollar el planteamiento anteriormente expuesto, se estimaron algunos objetivos por fase entre los cuales se encuentran:

Fase de acción. Provocar en los estudiantes la necesidad de la recolección de datos y el uso de instrumentos de medición del tiempo para que a partir de ello surja la construcción y la interpretación de gráficos estadísticos. Para determinar el estudiante con mayor resistencia física se propuso a los estudiantes realizar dos ejercicios físicos, el primero consistió en correr alrededor de la cancha del colegio cronometrando por equipos el tiempo de esquina a esquina y el segundo en realizar sentadillas durante 30 segundos.

Fase de Formulación. A partir de los hechos de clase los estudiantes proponen soluciones a la situación fundamental planteada. Con el ánimo de orientar las acciones de los estudiantes, durante ésta fase se construyeron grupos de trabajo en los que cada integrante presentó el resultado de su resistencia física con base en los dos ejercicios ejecutados a los compañeros del grupo. Posterior a ello cada subgrupo seleccionó al estudiante que tuviera mayor resistencia física.

Fase de validación. Se invita a la discusión grupal de los resultados y las razones que expone cada grupo para postular un ganador.

\section{Logros y dificultades evidenciadas}

La necesidad de medir el tiempo con un instrumento de precisión fue vista por los estudiantes al inicio del experimento, por ello se considera que desde ésta sencilla acción se potencia la selección de unidades estandarizadas, apropiadas para diferentes mediciones.

De manera posterior, se pretendía que cada estudiante con su propia información de rendimiento de acuerdo a los dos experimentos realizados, adquiriera mayores comprensiones sobre representaciones estadísticas, por ello, se propuso representar en gráficas cartesianas la información contenida en las tablas.

Algunos estudiantes tuvieron dificultades relacionadas con las reglas de la representación, entre ellas la ubicación del origen del plano cartesiano iniciando desde números distintos al cero lo cual sucede porque no se ha configurado la idea de número como representación de una distancia. Frente a esto Li \& Shen (citados por Batanero, 2001) rescatan algunos errores similares, entre los cuales están: omitir las escalas en alguno de los ejes horizontal o vertical, o en ambos, no especificar el 
origen de coordenadas y no proporcionar suficientes divisiones en las escalas de los ejes.

De manera reiterativa los estudiantes argumentaban que si se aumentaban el número de vueltas, conservando los mismos resultados durante el experimento, resultaba ganador aquel que le tomara menor tiempo recorrer la cancha. En los argumentos de los estudiantes se encuentra inmersa la idea de covariación entendida como la relación existente entre dos magnitudes o series estadísticas, de manera que todo aumento o disminución de una de ellas se traduce en un aumento o disminución de la otra.

En cuanto a las variables cualitativas, los estudiantes señalaron que existían variaciones en la resistencia física determinadas por el cansancio, la fatiga y la instrumentación no adecuada.

Finalmente y considerando que el aprendizaje desde la TSD es efectivo cuando el estudiante cambia de contexto y da respuesta a los mismos elementos conceptuales, (Panizza, 2004), en el proceso final de evaluación, se presentó a los estudiantes tres contextos (venta de helados, de cometas y juguetes) a partir de los cuales se evidenció en los estudiantes por un lado, la realización de análisis cuantitativo a partir de la interpretación y transnumeración de gráficos estadísticos simples y a nivel cualitativo, se encontró que los niños nombraron diferentes variables que podrían afectar la venta de cometas, helados y juguetes, entre ellas la época del año, el clima y el gusto actual de los niños por artículos electrónicos respectivamente.

\section{Reflexiones finales}

A partir de lo denominado por Wild \& Pfannkuch (citados por Batanero, 2003) como transnumeración, se considera que el grupo se encuentra en la capacidad de construir y realizar lectura literal de gráficos estadísticos e inferencias ligadas a los datos relacionados en éstas, siempre y cuando se mantenga una regularidad en términos de crecimiento y decrecimiento visto a partir de gráficos de barras y gráficos cartesianos.
Desde dichas observaciones vale la pena señalar que en el proceso de transnumeración visto para estudiantes próximos al ingreso a bachillerato, no sólo influye el cambio de representación y la interpretación de la información escrita, también están relacionadas las reglas fundamentales de la representación, es decir como desde la simbología de los gráficos cartesianos y de barras se representa la dependencia de una variable respecto a otra, se configura la idea de número como magnitud, entre otros.

En cuanto a las variables cualitativas, se considera que los niños pueden caracterizar situaciones en contextos cercanos a sus vivencias personales y a partir de ello establecer variables no relacionadas en gráficos estadísticos o tablas que representen una situación.

\section{Referencias}

Arellano, B. (2001). Estadística y Probabilidad. México: Editorial Cengage Learning Latin America.

Batanero, C. (2001). Didáctica de la Estadística. España: Grupo de educación estadística Universidad de Granada.

Batanero, C. (2003). Los retos de la cultura estadística. Congreso de Estadística. Recuperado de http://www.docentes.unal.edu.co/pnpachecod/docs/losretos.pdf.

Ministerio de Educación Nacional. (2006). Estándares básicos de competencias en Matemáticas. Recuperado de http://www.eduteka.org/pdfdir/ MENEstandaresMatematicas2003.pdf

Ministerio de Educación Nacional. (1998). Matemáticas. Lineamientos Curriculares. Recuperado de http://www.mineducacion. gov.co/cvn/1665/articles-89869_archivo_ pdf9.pdf.

Panizza, M. (2004). Conceptos básicos de la teoría de situaciones didácticas. Buenos Aires: Editorial Paidós. 